\title{
Searches for new phenomena in multilepton final states with the ATLAS and CMS detectors
}

Shilpi Jain*†

National Central University, Taiwan

E-mail: shilpi.jain@cern.ch

Hints of new physics in B-physics sector have lead to considerable interests on the flavor physics and lepton flavor violating observables at the LHC. Searches for lepton flavor violating decays of the Standard Model particles or new heavy particles have been conducted at the ATLAS and CMS experiment. This talk summarizes Run 1 searches for lepton flavor violating phenomena and new physics in multi-lepton/photon final states.

The European Physical Society Conference on High Energy Physics

22-29 July 2015

Vienna, Austria

* Speaker.

${ }^{\dagger}$ On behalf of the CMS and ATLAS collaborations 


\section{Introduction}

The success of Run I of LHC is marked by the discovery of the Higgs boson which was predicted in three papers written independently in 1964. The theory which predicted the existence of Higgs boson, known as the Brout-Englert-Higgs mechanism, was proposed by Francois Englert with Robert Brout [1], Tom Kibble with Gerald Guralnik and Richard Hagen [2, 3], and Peter Higgs $[4,5,6]$. The properties of the observed Higgs boson are like the ones which are predicted within the Standard Model (SM) of particle physics. Still there are many questions which are unanswered by the SM, for e.g., we do not yet know the origin of dark matter, if the quarks and leptons are fundamental, neutrino oscillation, etc. Observation of neutrino oscillation makes the study of lepton flavour violating (LVF) sector interesting. Multi-lepton and multi-photon final states are rich probes to look for new physics beyond the SM. There are various processes which can be studied in multi-lepton and multi-photon final states. This paper focuses on few of those processes, which includes the following:

1. Study of LVF in decays of $Z$ and new heavy resonances

2. Compositeness in the lepton sector

3. Type III seesaw models

4. New phenomena with multi-photon final state

\section{Search for LVF decays}

In spite of the absence of an underlying symmetry, lepton flavor is explicitly conserved in the SM. The observation of neutrino oscillation [7] in the neutral sector motivates the search for lepton flavour violation in the charged sector as well. If LVF in charged sector is observed, it would be a clear indication of new physics beyond the SM. There are other many new physics scenarios in which such signatures can occur, for e.g., R-parity violating SUSY, models with an additional heavy neutral gauge boson $Z^{\prime}$ etc. The first place to observe LVF decays is in $Z \rightarrow e \mu$ process. One can observe the LVF signature in not yet observed heavy resonances. Both CMS [8] and ATLAS [9] look for signature of LVF in decays of $Z$ and heavy resonances.

\subsection{LVF in $Z$ decays}

The branching fraction of $Z \rightarrow e \mu$ is predicted to be negligibly small [10] via one-loop decays with flavour-oscillating neutrinos, thus rendering its detection impossible in any near future experiment. Many new physics models, for e.g., Dirac or Majorana neutrinos, R-parity violating SUSY can increase the branching fraction of lepton flavour violating $\mathrm{Z}$ decays which can be detected at LHC [10, 11]. Both ATLAS [12] and CMS [13] searched for oppositely charged $e \mu$ pairs consistent with the $Z$ mass. Dominant background to this analysis is $Z \rightarrow \tau \tau$. CMS uses data-driven techniques to estimate the backgrounds, whereas, ATLAS estimated the background by fitting the data excluding the $Z$ region and then extrapolating it in the $Z$ region. With this analysis, CMS places a limit of $7 \times 10^{-7}$ and ATLAS places a limit of $7.5 \times 10^{-7}$ on the branching ratio of $Z \rightarrow e \mu$ which is one order of magnitude better than the results from LEP [14]. 


\subsection{LVF decays of heavy resonances}

As already mentioned above, new physics scenarios can increase the branching ratio of LVF decays. Such models predict the existence of a heavy neutral gauge boson $Z^{\prime}$ [15] allowing for LVF decays. Both ATLAS [16] and CMS [17] search for LVF in broad range spectrum of $\ell \ell^{\prime}$, where $\ell \ell^{\prime}$ is $e \mu$ in case of CMS and $e \mu, e \tau$ and $\mu \tau$ in case of search in ATLAS. Since ATLAS searches for LVF decays in the final state of $\tau_{\text {had }}$ which has missing transverse momentum in the final state, it becomes tough to reconstruct the mass of the heavy resonance. To reconstruct the four momentum vector of $v_{\tau}$, collinear neutrino approximation is used where $v$ and jets are assumed to be collinear (based on the fact that if heavy resonance exists, then $\tau$ is coming from the decay of a heavy resonance). This technique significantly improves the mass resolution. This way following are the limits from LHC:

1. ATLAS puts a limit of $2.0 \mathrm{TeV}$ on the invariant mass of $\tilde{v_{\tau}}$ in RPV SUSY. This is for $\Lambda_{311}^{\prime}=$ 0.11 and $\Lambda_{132}=0.07$. From CMS it is $1.3 \mathrm{TeV}$ for $\Lambda_{311}^{\prime}=0.01$ and $\Lambda_{132}=0.01$.

2. ATLAS puts a limit of $2.5 \mathrm{TeV}$ on the mass of $Z^{\prime}$ in SSM.

3. CMS puts a limit of $3.6 \mathrm{TeV}$ on $M_{t h}$ in Quantum black hole production.

\section{Search for excited leptons}

SM of particle physics does not provide any explanation for three generations of fermion families. There are many physics models which attempt to explain the observed hierarchy of fermions. One of such family of models which try to explain this observed phenomena by postulating that the observed quarks and leptons are composite objects of fundamental constituents [18, 19, 20,21]. Such models predict the existence of excited states of quarks $\left(q^{*}\right)$ and leptons $\left(\ell^{*}\right)$ at a characteristic scale $\Lambda$. Since such excited states couple to the SM fermions, these can be produced and further decay via contact interactions to other fermions or via gauge interactions to a SM fermion and a SM gauge boson. Both ATLAS [22] and CMS [23] searched for excited electrons and muons in Run I produced via contact interactions and decay via gauge interactions. ATLAS looked for the production of $\ell^{*}$ along with SM $\ell$ and its decay further to a SM $\ell$ and a $\gamma$ giving a final state of $\ell \ell \gamma$ (where $\ell=e, \mu$ ), whereas, CMS looked for a wider spectrum of final states: $\ell \ell \gamma$ (where $\ell=e, \mu$ ) (which is the same as ATLAS), $2 \ell 2 \ell^{\prime}$ ( $\ell^{*}$ produced along with a SM $\ell$ and then decaying to a SM $\ell$ and a $Z$, with $Z$ decaying to either $2 e^{\prime} \mathrm{s}$ or $2 \mu^{\prime} \mathrm{s}$ ). This gives a final state of $4 e^{\prime} \mathrm{s}$ or $4 \mu^{\prime} \mathrm{s}$ or $2 e^{\prime} \mathrm{s}$ $2 \mu$ s. CMS also studied the case where $Z$ decays to 2 jets. In case of CMS, for the decay mode $\ell^{*} \rightarrow \ell Z$, the decay products from $Z$ are collinear since $Z$ is boosted (it being coming from decay of a heavy particle). In such a case, applying the standard isolation in calorimeter or tracker around the decayed product (e.g. $e$ ) would reduce the signal efficiency by a large factor. To maintain the efficiency in this case, relaxed isolation is applied. To search for $\ell^{*}$, ATLAS constructs a 3 body invariant mass $\left(\mathrm{M}_{\ell \ell \gamma}\right)$ and performs a cut and count analysis. Whereas, in case of CMS following is done for the two different cases:

1. $\ell \ell \gamma$ final state: Since there are two same flavour leptons in the final state without the knowledge of which lepton comes from the decay of $\ell^{*}$, two invariant masses are possible $\left(\mathbf{M}_{\ell \gamma}^{M a x}\right.$ 
and $\mathbf{M}_{\ell \gamma}^{M i n}$ ). Since one of the combinations is right (for an event), a 2-D scatter plot of $\mathbf{M}_{\ell \gamma}^{M a x}$ and $\mathrm{M}_{\ell \gamma}^{\text {Min }}$ gives an inverted "L-shape" feature for the signal, whereas, the background lies flat in this 2-D space. In CMS, such an "L-shape" is feature is exploited for this search. For a particular signal mass point, a 2-D region inverted "L-shape" region is formed around the mass hypothesis and that region is scanned for any excess above the SM expectation.

2. $2 \ell \mathbf{2} \ell^{\prime}$ final state: Once the $Z$ is reconstructed, one again is left with two same flavour leptons in the final state without the knowledge of which lepton comes from the decay of $\ell^{*}$. Here also (as in the case of $\ell \ell \gamma$ ) two invariant masses are possible $\left(\mathbf{M}_{\ell \gamma}^{M a x}\right.$ and $\mathbf{M}_{\ell \gamma}^{M i n}$ ). Exactly the same procedure is followed as above by scanning the 2-D region of $\mathbf{M}_{\ell \gamma}^{M a x}$ and $\mathbf{M}_{\ell \gamma}^{\mathrm{Min}}$ for any excess above the SM expectation.

Figure 1 shows the 2-D scatter plot $\mathrm{M}_{\ell \gamma}^{\mathrm{Max}}$ and $\mathrm{M}_{\ell \gamma}^{\mathrm{Min}}$ used in the excited lepton analysis in CMS. It also shows the 3-body invariant mass used for search in ATLAS.

In CMS also, cut and count analysis is performed. Both ATLAS and CMS did not see any excess above the SM expectation and limits are placed on the mass of excited lepton. ATLAS excludes massed below $2.2 \mathrm{TeV}$, whereas, CMS has the following limits:

1. For $f=f^{\prime}$ in $\ell \ell \gamma$ final state: masses below $2.45(2.48) \mathrm{TeV}$ are excluded in $e(\mu)$ channel.

2. For $f=-f^{\prime}$ in $\ell \ell Z$ final state: masses below $2.35(2.38) \mathrm{TeV}$ are excluded in $e(\mu)$ channel.

\section{Heavy-lepton partners of neutrinos (Type III seesaw)}

Observation of neutrino oscillation is a direct indication that neutrinos have mass [24]. There are many models which can explain this observation. Among them is the seesaw mechanism which introduces new heavy particles that couple both to the leptons and to the Higgs doublets, thus accounting for both the neutrino masses and their smallness. These new heavy particles could be weak-singlet fermions ([25, 26, 27, 28]); weak-triplet scalars ([29, 30, 31, 32, 33]); or weak-triplet fermions ([34]). ATLAS [35] and CMS [36] have searched for type III heavy-lepton partners of neutrinos $\left(\Sigma^{+}, \Sigma^{0}, \Sigma^{-}\right)$which can be produced through quark-annihilation via a virtual boson as follows: $q \bar{q} \rightarrow W^{ \pm} \rightarrow \Sigma^{0} \Sigma^{ \pm}$and $q \bar{q} \rightarrow Z \rightarrow \Sigma^{+} \Sigma^{-}$. This is further followed by the decay of heavy fermions to SM bosons and leptons. CMS searches for a final state of 3 leptons and MET, whereas, ATLAS looks for 2 isolated leptons and at least 2 jets and MET. Parameters of the theory are $V_{e}, V_{\mu}, V_{\tau}$ which are the mixing angles between the SM and the triplet state. With this analysis, ATLAS excludes mass of the heavy resonance below $335 \mathrm{GeV}$ for $V_{\tau}=0$ and $V_{e} / V_{\mu}=0.87$, whereas, CMS excludes masses below $280 \mathrm{GeV}$ for $V_{e}=V_{\mu}=V_{\tau}=10^{-6}$. Assuming only $\tau-e$ mixing, CMS excludes mass of heavy resonance below $320 \mathrm{GeV}$.

\section{New phenomena with multi-photon}

There are many new physics scenarios (essentially extensions of SM) that can result in final state of three or more photons. Following are some of the models which predict a final state of 3 or more photons: 

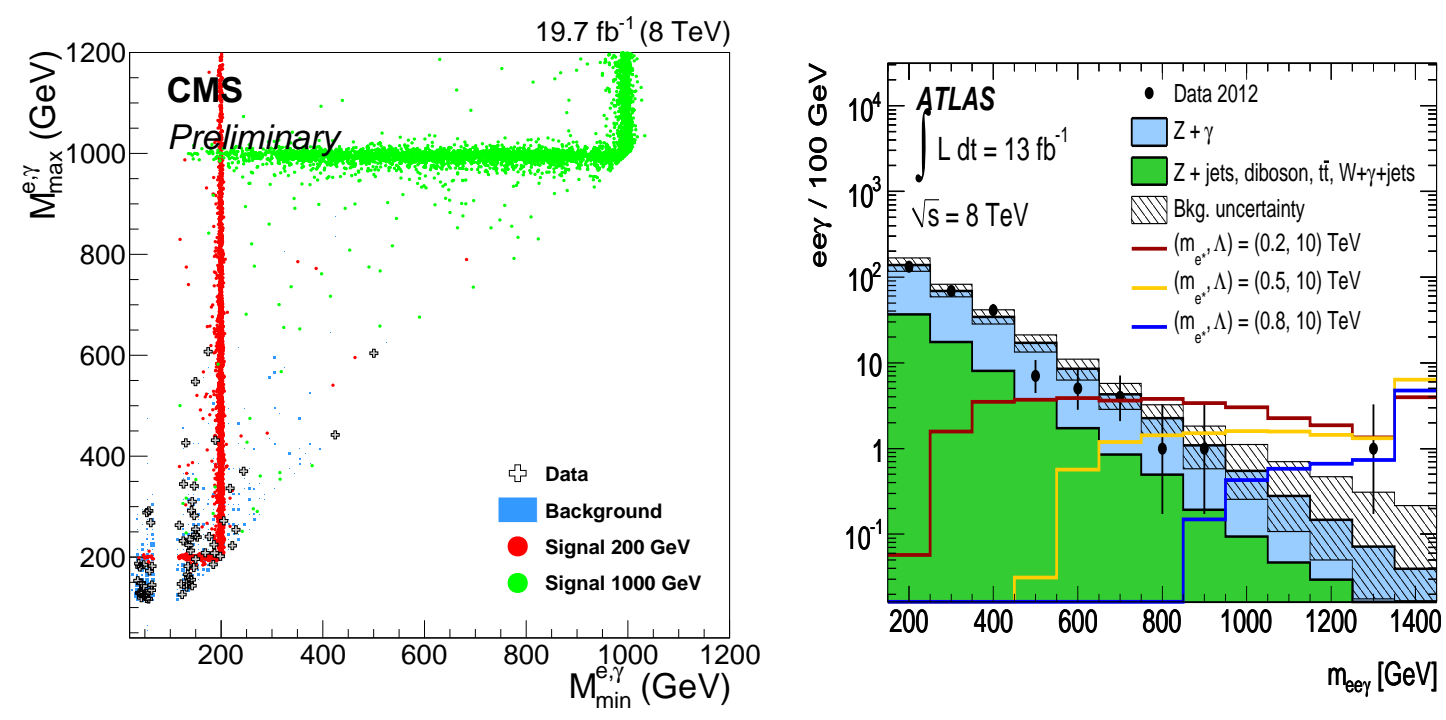

Figure 1: Plot on the left shows the $M_{\ell \gamma}^{M a x}$ VS $M_{\ell \gamma}^{M i n}$ used in the excited lepton analysis. Once can see the "inverted L-shape" feature in the signal [23]. Plot on the right shows the 3-body invariant mass used for excited lepton search in ATLAS [22].

1. Models which predict pseudo-scalar particles (a) with couplings to the Higgs boson $(h)$ which can further decay to photons ( $h \rightarrow a a \rightarrow 4 \gamma$ ). Such models can have coupling of $a$ with scalars which have masses different from the SM Higgs boson $(H)$ that can also decay to 2 photons.

2. Models which have additional gauge bosons that can decay to a photon and a new pseudoscalar boson ( $a$ ), with the subsequent decay of $a$ into two photons, thus giving a final state of 3 photons.

3. In SM, $Z$ boson can decay into three photons via $W^{ \pm}$loop or fermion loop. This decay is negligibly small $\left(\sim 5 \times 10^{-10}\right)$ [37]. This branching ratio can be enhanced by the presence of some new physics.

Figure 2 shows the Feynman diagrams of such processes. ATLAS performed a search in events with at least three photons using $20.3 \mathrm{fb}^{-} 1$ of LHC data at $8 \mathrm{TeV}$. It did not observe any excess 


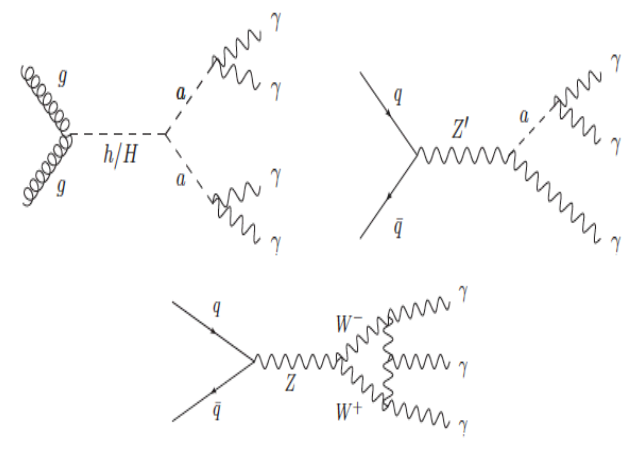

Figure 2: Feynman diagrams for possible beyond the SM scenarios which can result in multi-photon final state.

over the SM background and places a limit on the $B R(Z \rightarrow 3 \gamma)<2.2 \times 10^{-6}$ [38] which is one order of magnitude stringent than the previous limits from L3 $\left(<10^{-5}\right)[39]$.

\section{References}

[1] F. Englert, R. Brout, Broken symmetry and the mass of gauge vector mesons, Phys. Rev. Lett. (1964), 10.1103/PhysRevLett.13.321

[2] G.S. Guralnik, C. R. Hagen, T.W.B. Kibble, Global conservation laws and massless particles, Phys. Rev. Lett. (1964), 10.1103/PhysRevLett.13.585

[3] T.W.B. Kibble, Symmetry breaking in non-Abelian gauge theories, Phys. Rev. Lett. (1967), 10.1103/PhysRev.155.1554

[4] P.W. Higgs, Broken symmetries, massless particles and gauge fields, Phys. Rev. Lett. (1964), 10.1016/0031-9163(64)91136-9

[5] P.W. Higgs, Broken symmetries and the masses of gauge bosons, Phys. Rev. Lett. (1964), 10.1103/PhysRevLett.13.508

[6] P.W. Higgs, Spontaneous symmetry breakdown without massless bosons, Phys. Rev. Lett. (1964), 10.1103/PhysRev.145.1156

[7] SNO Collaboration, Direct evidence for neutrino flavor transformation from neutral current interactions in the Sudbury Neutrino Observatory, Phys. Rev. Lett. 89 (2002), doi:10.1103/PhysRevLett.89.011301, arXiv:nucl-ex/0204008

[8] CMS Collaboration, The CMS experiment at the CERN LHC, JINST 3:S08004 (2008)

[9] ATLAS Collaboration, The ATLAS experiment at the CERN LHC, JINST 3:S08003 (2008)

[10] J.I. Illana and T.Riemann, Charged lepton flavor violation from massive neutrinos in Z decays, Phys. Rev. D63 (2001), doi:10.1103/PhysRevD.63.053004, arXiv:hep-ph/0010193

[11] J.M. Yang, Lepton flavor violating Z-boson decays at GigaZ as a probe of supersymmetry, Sci.China G53 (2010), doi:10.1007/s11433-010-4146-3, arXiv:1006.2594 
[12] ATLAS Collaboration, Search for the lepton flavor violating decays $Z \rightarrow e \mu$ in pp collisions at $\sqrt{s}=8$ TeV with the ATLAS detector, Phys. Rev. D90 (2014), 10.1103/PhysRevD.90.072010

[13] CMS Collaboration, Search for Lepton Flavor Violation in Z decays in pp collisions at $\sqrt{s}=8 \mathrm{TeV}$, CMS-PAS-EXO-13-005

[14] OPAL Collaboration, A Search for lepton flavour Violating $Z^{0}$ decays , Z.Phys. (1995), C67, doi:10.1007/BF01553981

[15] P. Langacker, Rev. Mod. Phys.(2009), arXiv:0801.1345

[16] ATLAS Collaboration, Search for a Heavy Neutral Particle Decaying to $e \mu$, $e \tau$, or $\mu \tau$ in $p p$ Collisions at $\sqrt{s}=8 \mathrm{TeV}$ with the ATLAS Detector, Phys. Rev. Lett. (2014), 10.1103/PhysRevLett.115.031801

[17] CMS Collaboration, Search for Lepton Flavour Violating Decays of Heavy Resonances and Quantum Black Holes to electron/muon Pairs in pp Collisions at a centre of mass energy of $8 \mathrm{TeV}$, CMS-PAS-EXO-13-002

[18] J.C. Pati, A. Salam, and J.A. Strathdee, Are quarks composite?, Phys. Lett. B 59(1975) 265, doi:10.1016/0370-2693(75)90042-8

[19] H. Terazawa, M. Yasue, K. Akama, and M. Hayshi, Observable effects of the possible substructure of leptons and quarks, Phys. Lett. B 112(1982) 387, doi:10.1016/0370-2693(82)91075-9

[20] H. Harari, Composite models for quarks and leptons, Physics Reports 104(1984) 159, doi:10.1016/0370-1573(84)90207-2

[21] U. Baur, M. Spira, and P.M. Zerwas, Composite models of Leptons, Phys. Rev. D 10(1974) 2567 , doi:10.1103/PhysRevD.10.2567

[22] ATLAS Collaboration, Search for excited electrons and muons in $\sqrt{s}=8 \mathrm{TeV}$ proton-proton collisions with the ATLAS detector, New Journal of Physics 15 (2013), doi:10.1088/1367-2630/15/9/093011

[23] CMS Collaboration, Search for excited leptons in proton proton collisions at $\sqrt{s}=8 \mathrm{TeV}$, CMS-PAS-EXO-14-015

[24] F. Capozzi et al., Status of three-neutrino oscillation parameters, circa 2013, Phys. Rev. D 89(2014), arXiv:1312.2878

[25] T. Han and B. Zhang, Signatures for Majorana neutrinos at hadron colliders, Phys. Rev. Lett. 97(2006), doi:10.1103/PhysRevLett.97.171804

[26] P. Minkowski, $\mu \rightarrow$ e $\gamma$ at a rate of one out of $10^{9}$ muon decays?, Phys. Rev. Lett. 67(1977) 421, doi:10.1016/0370-2693(77)90435-X

[27] R. Mohapatra, N. Rabindra, and G. Senjanovic, Neutrino Mass and Spontaneous Parity Violation, Phys. Rev. Lett. 44(1980) 912, doi:10.1103/PhysRevLett.44.912

[28] T. Yanagida, Neutrino Mass in seesaw type I, Proc. of the Workshop on Unified Theory and Baryon Number of the Universe 64(1979) 1103

[29] M. Magg and C. Wetterich, Neutrino Mass Problem and Gauge Hierarchy, Phys. Rev. B 94(1980) 61, doi:10.1016/0370-2693(80)90825-4

[30] J. Schechter and J. Valle, Neutrino masses in SU(2)xU(1) theories, Phys. Rev. D 22(1980) 2227 , doi:10.1103/PhysRevD.22.2227 
[31] C. Wetterich, Neutrino Masses and the Scale of B-L Violation, Nucl. Phys. B 187(1981) 343, doi:10.1016/0550-3213(81)90279-0

[32] G. Lazarides, Q. Shafi, and C. Wetterich, Proton Lifetime and Fermion Masses in an SO(10) Model, Nucl. Phys. B 181(1981) 287, doi:10.1016/0550-3213(81)90354-0

[33] R. Mohapatra and G. SEnjanovic, Neutrino Masses and Mixing in Gauge Models with Spontaneous Parity Violation, Nucl. Phys. D 23(1981) 165, doi:10.1103/PhysRevD.23.165

[34] R. Foot, H. Lew, X. He, and G. Joshi, See-saw neutrino masses induced by a triplet of leptons, Z. Phys. C 44(1989), doi:10.1007/BF01415558

[35] ATLAS collboration, Search for type-III seesaw heavy leptons in pp collisions at $\sqrt{s}=8 \mathrm{TeV}$ with the ATLAS detector, Phys. Rev. D 92(2015), 10.1103/PhysRevD.92.032001

[36] CMS collboration, Search for heavy lepton partners of neutrinos in pp collisions at $\sqrt{s}=8 \mathrm{TeV}$, in the context of type III seesaw mechanism, CMS-PAS-EXO-14-001

[37] E. N. Glover and A. Morgan, Z boson decay into photons, Z.Phys. C60(1993)

[38] ATLAS collaboration, Search for new phenomena in events with at least three photons collected in pp collisions at $\sqrt{s}=8 \mathrm{TeV}$ with the ATLAS detector, arXiv:1509.05051 [hep-ex], submitted to EPJC

[39] L3 Collaboration, Search for anomalous $Z \rightarrow \gamma \gamma \gamma$ events at LEP, Phys. Lett. B345 (1995) 609-616 